\title{
The functional equivalence of operant behavior and foraging
}

\author{
JESSE DALLERY and WILLIAM M. BAUM \\ University of New Hampshire, Durham, New Hampshire
}

\begin{abstract}
Many researchers have used the controlled procedures of operant methodology to test the predictions of foraging theory on the assumption that foraging is operant behavior and operant behavior is foraging. This experiment tested the assumption that leverpressing is functionally equivalent to search effort. Four rats were exposed to two situations: a naturalistic foraging situation (a "patch") and a standard operant chamber. Each condition represented a different prey density on the patch and a corresponding change in the probability of reinforcement in the chamber. The average number of presses per pellet was linearly related to the density of pellets on the patch. This result indicates that leverpresses were functionally equivalent to search effort.
\end{abstract}

Recently, operant psychologists have become interested in relating traditional learning experiments to foraging, and behavioral ecologists have increasingly been employing operant techniques and equipment as tools for the study of foraging (Abarca \& Fantino, 1982; Baum, 1983; Collier \& Rovee-Collier, 1981; Hanson, 1987; Hanson \& Green, 1989a, 1989b; Kamil, Krebs, \& Pulliam, 1987; Kamil \& Sargent, 1981; Lea, 1979; Schull, Gelch, Vitale, Allan, James, \& Harrison, 1985; see Shettleworth, 1988, for a review). In laboratory studies of learning and choice, in which operant behavior is reinforced with food, it can be said that animals "forage" for food. But does their behavior in the laboratory have any relationship to foraging in nature? Are keypecks or leverpresses equivalent to search effort?

Most experiments that have treated operant behavior as if it were foraging have sought to test optimal foraging theory (OFT). Generally, OFT predicts that animals, as a result of natural selection, will collect food in a way that maximizes their net rate of food intake (see Schoener, 1987 , for a review). In testing such predictions, ecologists have found it difficult to meet the simplifying assumptions of OFT (Krebs, Stephens, \& Sutherland, 1983). Some of these assumptions include: (1) Searching for and handling prey items are mutually exclusive activities; (2) individual prey items are encountered randomly and sequentially; (3) prey items are clearly discriminable and instantly recognizable; and (4) the forager has accurate knowledge of environmental parameters such as energy costs, handling times, search costs, encounter rates, and so forth.

This research was supported by Grant BNS 84-01119 from the National Science Foundation to William M. Baum and a grant from the Undergraduate Research Opportunities Program of the University of New Hampshire to Jesse Dallery. Requests for reprints should be addressed to William M. Baum, Department of Psychology, University of New Hampshire, Durham, NH 03824-3567.
In contrast to naturalistic tests of OFT, operant tests are uniquely suited to meet these simplifying assumptions:

1. If operant behavior on a certain schedule is analogous to searching, then it is mutually exclusive of handling, whether real (i.e., eating) or simulated (i.e., on another schedule; Abarca \& Fantino, 1982).

2. Individual prey items (i.e., reinforcers) are arranged randomly and sequentially in such schedules as variable ratio and variable interval.

3. Reinforcers are accompanied by visual and auditory cues that make them highly discriminable and instantly recognizable.

4. The procedural analogue to "accurate knowledge", is to repeat the operant procedure day after day until stable performance is achieved. The experimenter can manipulate costs, encounter rates, and prey discriminability from one procedure to the next.

It should be no surprise that operant tests of OFT are plentiful.

Early operant tests of OFT mimicked the foraging situation by using three schedule components: (1) search state; (2) choice state; and (3) handling state (e.g., Abarca \& Fantino, 1982; Lea, 1979). In Lea's (1979) experiment, the animal was exposed to a search state schedule in which pecks on a fixed-interval (FI) schedule led to the initiation of a choice state. The choice state consisted of the presentation of two differently colored keys. A single peck on one of the keys initiated the handling state. The handling state led to food according to an FI schedule that was correlated with a key illuminated by one of two colors. The differently colored keys represented either a rich (FI $5 \mathrm{sec}$ ) or a poor (FI $20 \mathrm{sec}$ ) prey type. Abarca and Fantino (1982) utilized a similar procedure, except that they used a variable-interval (VI) schedule in the handling state instead of an FI schedule.

These simulations have been criticized for their reliance on time-based schedules (Baum, 1983; Hanson, 1987; Hanson \& Green, 1989a, 1989b). Since the more a pred- 
ator hunts, the more prey it encounters, ratio schedules, which supply this sort of relation, seem more appropriate to model searching. Whether a forager is tracking a rodent, collecting nuts, or digging for worms, encounters with prey do not depend on the passage of time alone, but on the forager's efforts. Accordingly, some experimenters have used ratio schedules to model both searching and handling (e.g., Cheney, Bonem, \& Bonem, 1985; Hanson, 1987; Hanson \& Green, 1989a, 1989b).

If a resource depletes as it is exploited, then continued exploitation becomes ever more effortful. To model increasing effortfulness of discovering prey as predation progresses, some experiments have incorporated progressiveratio schedules (e.g., Cheney et al., 1985; Hanson, 1987; Hanson \& Green, 1989b) - that is, after each food delivery on the schedule, the work required for the next food delivery was increased. (See also Redhead \& Tyler, 1988, for an experiment with progressive-interval schedules.)

These experiments have generally produced results that accord with the predictions of OFT. This has encouraged the endorsement of operant methodology as a valid tool for the study of naturalistic foraging. Abarca and Fantino (1982) concluded that their results "encourage the use of technology developed in the operant laboratory in the study of foraging behavior" (p. 123), and Hanson (1987) stated that "many have noted that the methodology of operant psychology may be fruitfully applied to the foraging problem"' (p. 337).

This endorsement of operant methods may be premature. Collier and Rovee-Collier (1981) question whether we can substitute arbitrary responses for species-specific foraging responses. Different types of activities may lead to different experimental results (Hinde \& Stevenson-Hinde, 1973). Experimenters have used leverpressing, keypecking, perch-hopping (Ydenberg, 1984), and even remaining in a location (Cassini, Kacelnik, \& Segura, 1990) as if they were equivalent to the activities involved in foraging.

If they were functionally equivalent, then variation in any independent variable, such as effort required per prey item (reinforcer), that affected the time spent foraging would affect the amount of operant behavior in exactly the same way. Conversely, a procedure that matched times spent foraging and in operant responding ought to require, ceteris paribus, equivalent levels of the independent variable. Since this idea remains unsubstantiated, the present experiment was designed as a test.

Three requirements must be met in order to equate operant behavior with foraging. First, the presentation of food (i.e., reinforcement) must be analogous to the predator's encountering an item of prey. This can be achieved by using the same prey item (e.g., a food pellet) in both the natural environment and in the operant chamber. Second, for an operant model of foraging to be ecologically valid, the schedule on which the operant behavior produces food must resemble the schedule on which foraging produces prey in nature (e.g., a variable-ratio schedule; Baum, 1989). Finally, the operant behavior required for rein- forcement must be equivalent to the activities involved in foraging, such as search effort (Hinde \& StevensonHinde, 1973). The present experiment, by satisfying the first and second requirements, sought to test whether leverpressing meets the third requirement by comparing the effects of varying work effort in two situations: a standard operant chamber and a foraging apparatus resembling a patch of meadow over which small prey items such as seeds are scattered, a type of situation that rats might encounter in the wild.

\section{METHOD}

\section{Subjects}

Subjects were 4 male hooded rats bred at the University of New Hampshire and approximately 120 days of age at the beginning of the experiment. They were housed in individual cages in a colony room in which the lights were dim from 1200 to $0000 \mathrm{~h}$ and bright from 0000 to $1200 \mathrm{~h}$. The rats were maintained at $80 \%$ of their free-feeding body weight. Water was freely available in the home cage.

\section{Apparatus}

The seminaturalistic foraging apparatus ("patch") was a grid of 6,084 square holes placed on glass $165 \mathrm{~cm}$ above the floor in a small, windowless room. The patch measured $1.1 \times 1.1 \mathrm{~m}$. Each square hole in the patch measured $15 \times 15 \mathrm{~mm}$ with a depth of $10 \mathrm{~mm}$; the plastic separating adjacent holes was $2 \mathrm{~mm}$ thick. White noise was used to mask extraneous sounds. The rats' foraging behavior (i.e., capture rate and time taken) was observed through a monitor in the next room that was connected to a video camera underneath the patch. The experimenter recorded the time of each capture by pressing a key on the keyboard of a computer.

The operant chamber measured $25.5 \times 27.5 \mathrm{~cm}$, with one response lever available; this area was partitioned out of a larger wooden box. Three $28-\mathrm{V}$ dc lights were located on each side of the box. Events in the chamber were controlled and recorded by a microprocessor. The response lever was $85 \mathrm{~mm}$ from the left wall and $23 \mathrm{~mm}$ from the floor and required a minimum force equivalent to a weight of $62 \mathrm{~g}$ to operate a microswitch. Single food pellets were dispensed into a cup ( $32 \times 29 \mathrm{~mm}, 12 \mathrm{~mm}$ deep) $3.5 \mathrm{~cm}$ from the lever. The prey on the patch and the reinforcers in the chamber consisted of 45-mg grain-based food pellets (P. J. Noyes Co., "Formula A").

\section{Procedure}

Initial training on the patch consisted of placing a rat on the patch with a small pile of pellets in the center. After the rat fed without hesitation on the patch, a large number of pellets was distributed over the patch. Each rat had received about 30 such sessions when the first condition was begun. In the chamber, the rats were trained to press the lever by hand shaping. Each rat had received about 20 sessions of pressing on a random-ratio schedule with a probability of reinforcement of 0.8 when the first condition was begun.

Sessions were conducted in pairs, one session on the patch and one in the chamber. For the first pair of sessions of a condition, the session on the patch preceded the session in the chamber. After the first pair of sessions, the order was the reverse, operant chamber first and then patch.

Prior to each session on the patch, a certain number of pellets (see Table 1) was distributed irregularly by hand over the patch. Half of the pellets were distributed over half of the patch, and then the other half of the pellets were distributed over the other half of the patch. A hole was allowed to contain, at most, one pellet. This 
method was intended to result in an approximately random distribution over the patch. To test whether the distribution was random, one experimenter (J.D.) distributed 90 pellets over the patch, and the other experimenter (W.M.B.), without communicating with J.D., divided the patch into nine equal squares and counted the number of pellets in each square to obtain a frequency distribution. This was done five times, and each frequency distribution was tested for deviation from randomness by a chi-squared test. Neither the individual distributions nor their average differed significantly from randomness $(p>.3)$.

The patch session was terminated after the rat ate $60 \%$ of the pellets available. The total number of pellets obtainable in the chamber was set equal to the number of pellets eaten on the patch. (For example, if 30 pellet captures terminated the patch session, then 30 reinforcers would terminate the operant session.)

After placing a rat in the operant chamber, the experimenter waited until he heard at least one press on the lever before starting the session. This insured that the rat was at the lever when the session began. The starting of the session turned on all six lights in the chamber, after which the first press extinguished the lights on the left; the three on the right remained on until the delivery of the last reinforcer.

The schedule in the operant chamber was a random-ratio schedule. For the first operant session of each condition, the experimenter (J.D.) guessed at a probability of reinforcement that would equate the time in the chamber with the time on the patch. For the remainder of the condition, the random-ratio schedule was set by a titration procedure: If the difference between the durations of the operant and patch sessions in a pair exceeded $10 \%$ of the duration of the patch session, then the probability of reinforcement in the next operant session was increased or decreased by .05 . This resulted in the approximate equality of the durations of the two types of sessions by the end of each condition.

The procedure was analogous to a psychophysical matching task in which an independent variable such as luminance (here, probability of reinforcement) is adjusted until a dependent variable such as brightness (here, time spent responding) is matched across two situations. The dependent variable of the experiment thus becomes the level of the adjusted independent variable (probability of reinforcement) required to match one situation to the other. Only one situation is adjusted; the other (here, the patch) is considered the standard and is changed only across conditions.

The procedure for continuous reinforcement (FR 1) was a bit different. Instead of varying the probability of reinforcement, which was fixed at 1.0 , the number of pellets available on the patch was varied. If the durations differed by $10 \%$ or more, then the number of pellets placed on the patch was increased or decreased by 20 . The number of pellets to terminate the sessions was always $60 \%$ of the number available on the patch.

Table 1

Summary of Conditions, Showing Order, Number of Pellets Available on the Patch, and Number of Session Pairs on the Patch and in the Operant Chamber

\begin{tabular}{ccc}
\hline Condition & No. Pellets & No. Session Pairs \\
\hline 1 & 50 & 21 \\
2 & 25 & 7 \\
3 & 15 & 7 \\
4 & 75 & 11 \\
5 & FR 1 * & 7 \\
6 & 50 & 9 \\
7 & 15 & 7
\end{tabular}

*FR 1:

Rat 1

120

Rats 2 and 3

100

Rat 4

80
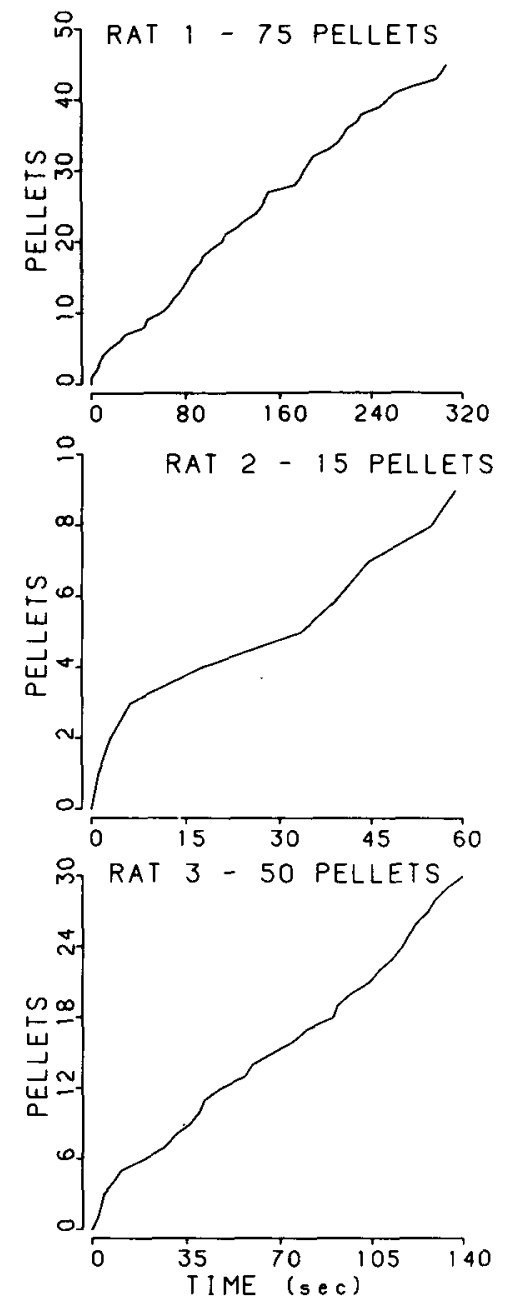

Figure 1. Cumulative records of number of pellets eaten by 3 rats at three different prey densities.

With rare exceptions, sessions were conducted 7 days a week. Usually one session was conducted per day. Sometimes two sessions were conducted on the same day, always at least $2 \mathrm{~h}$ apart. This appeared to have no effect on the results. The rats were always fed in their home cages at least $2 \mathrm{~h}$ after the last session of the day.

Each condition lasted for a minimum of seven pairs of sessions. After seven pairs, the durations of the last five sessions of each type were averaged each day for each rat. The condition continued until, for all 4 rats, the durations appeared stable by inspection and the two average durations differed by less than $10 \%$; then the next condition was begun. Table 1 shows the order of conditions and the number of sessions for which each lasted.

\section{RESULTS}

The data were summarized by averaging the durations and numbers of leverpresses across the last five sessions in each condition. (For FR 1, the numbers of pellets available were averaged.)

One common assumption of foraging theories appeared to be met in this study: Observation indicated that the rats 
spent all their time either searching and handling pellets on the patch or leverpressing and handling pellets in the chamber. The amount of time spent in extraneous behavior such as grooming or rearing appeared negligible.

Figure 1 shows cumulative records of number of pellets captured as a function of time spent on the patch for three representative sessions at three prey densities. Apart from the first one or two pellets of a session, the records are all approximately linear, indicating an approximately constant capture rate. The times of the first one or two captures were subject to a small artifact, because the experimenter had to run from one room to the other to begin recording. Since the rats began searching immediately, the time to the first capture necessarily fell short by the time required to change rooms, and if a capture had already occurred by the time recording began, the time of the second capture was curtailed as well. These approximately linear records resemble those produced by variable-ratio schedules (Ferster \& Skinner, 1957).

Figure 2 shows the average number of leverpresses per pellet as a function of the reciprocal of the number of pellets available on the patch, which was directly proportional to average area searched per pellet. Although normally an independent variable, presses per pellet is a dependent variable here, because it is the outcome of a procedure that matched time spent responding in the two situations. As in a psychophysical matching task, the dependent variable is the level of the independent variable necessary to achieve equal effects. Each point represents the results from one condition in Table 1 . The closeness of points lying one directly above the other indicates that results were readily replicated. The one exception was the high outlier for Rat 4, which represents the very first condition. Since experiments on operant behavior commonly produce aberrant results in the initial conditions, results that fail to be repeated when the conditions are reinstated, the outlier was attributed to inexperience and disregarded. The lines were fitted by the method of least squares. Correlation coefficients for Rats 1 through 4 were $0.96,1.0,0.99$, and 1.0. For all rats, the function between leverpressing and search effort was approximately linear.

Figure 3 shows the average capture rate on the patch as a function of the number of pellets available. For all rats, the capture rate increased as the number of pellets available on the foraging apparatus increased. The curve fitted to the points is derived from a version of Holling's (1959) "disc equation":

$$
r=\frac{1}{t+H}
$$

where $t$ is the search time required to encounter a prey item and $H$ is the handling time; if $t+H$ is the total time spent per prey item, then Equation 1 states that the capture rate is simply the reciprocal of the average time spent per prey item. Since $H$ is assumed to depend only on the type of prey and to be independent of density, variation in $r$ is assumed to result from variation in $t$, but $t$ is as-
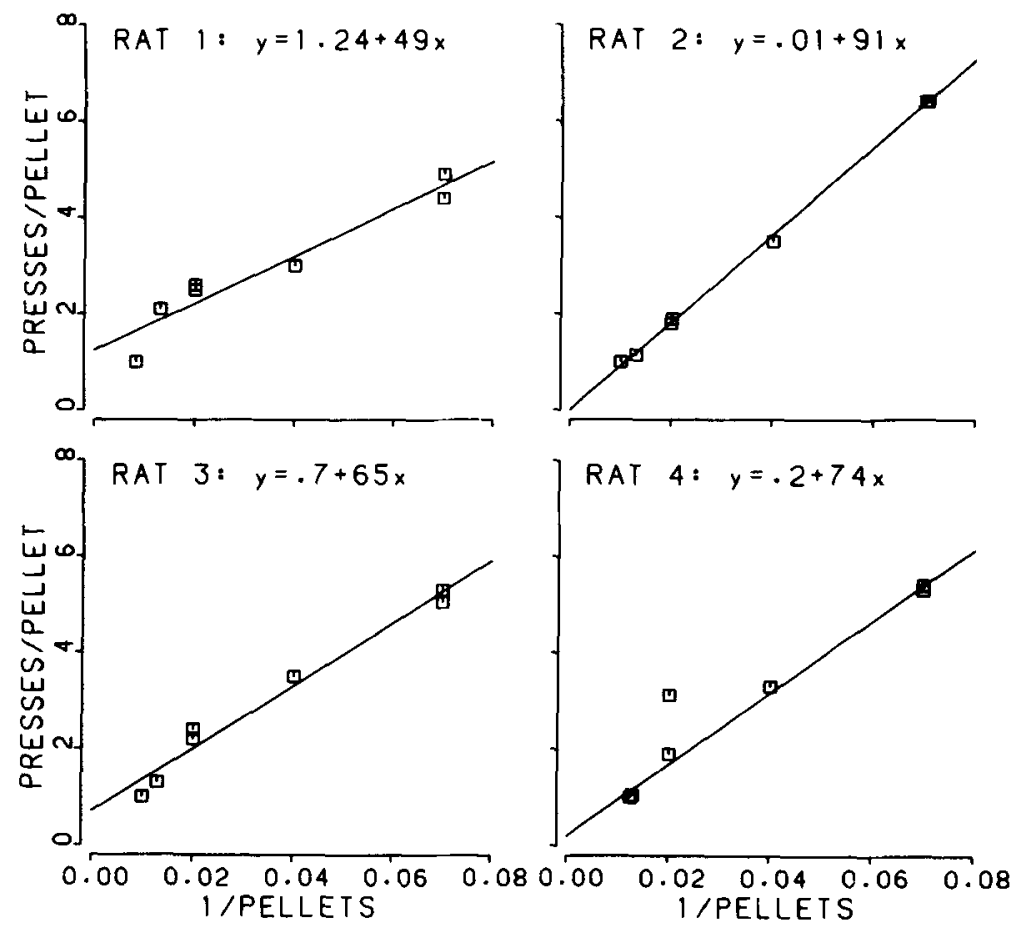

Figure 2. Average number of leverpresses per pellet as a function of the reciprocal of the number of pellets available. The lines (equations shown) were fitted by the leastsquares method. 

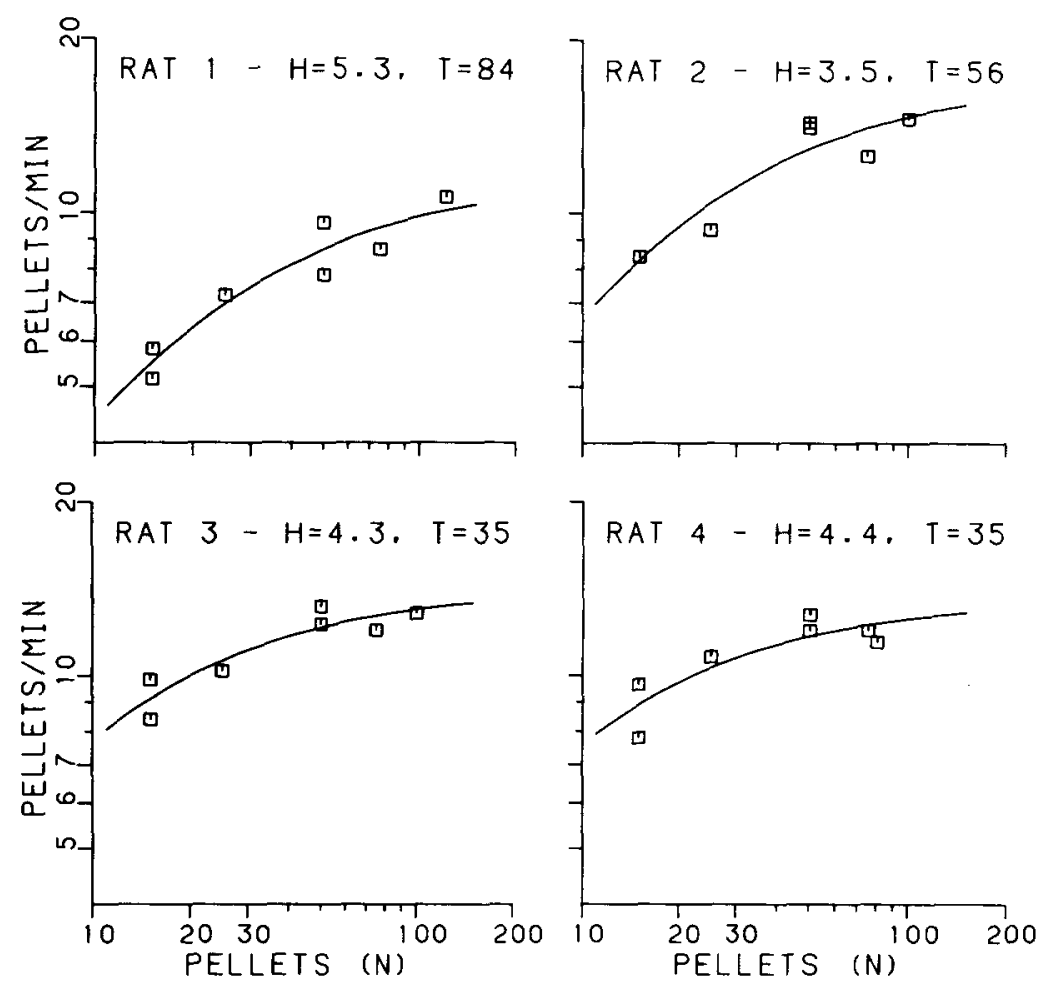

Figure 3. Average capture rate on the patch as a function of the number of pellets available. The curves indicate the best fit of Equation 3, a version of Holling's (1959) disc equation; the values of $H$ at $T$ are shown.

sumed fixed for a given condition and to vary only across conditions. Substituting $1 / D S$ for $t$, where $D$ is prey density (prey per unit of area) and $S$ is search rate (area per unit of time), on the assumption that the rate of encounter $D S$ remains fixed throughout, as implied by the linearity of the records in Figure 1, we obtain:

$$
r=\frac{D S}{H D S+1} \text {. }
$$

Substituting $N / A$ for $D$, where $N$ is the number of pellets available on the patch and $A$ is the area of the patch, and rearranging, we obtain:

$$
r=\frac{N}{H N+T}
$$

where $T$ is equal to $A / S$, the time that would be required to search the entire patch. The curves in Figure 3 represent the best fits of Equation 3 by the method of least squares. The fits were generally good; the proportions of variance accounted for ranged from .77 to .90 . The best-fitting values of $H$ and $T$ are given for each rat.

The results for FR 1 generally accord with the results from the other conditions. The one possible exception is the low (leftmost) point in Figure 2 for Rat 1. The number of pellets available for this rat (120) exceeded that for any other rat (Table 1). It is possible that this density was high enough to reduce search time to virtually zero.
Since the estimated handling time on the patch was greater for Rat 1 than for any other, and the observed capture rate of 10.6 was close to the theoretical maximum (the asymptote of Equation 3,11.3), it seems possible that virtually all its time was spent handling pellets (i.e., fishing them out of holes and eating them). If so, then it would have made no difference if there were 120 pellets or an infinite number of pellets available, and the point could have been positioned horizontally anywhere between its present position and the $y$-axis. If it were close to the $y$-axis, it would no longer appear aberrant.

\section{DISCUSSION}

How do the linear relations in Figure 2 imply functional equivalence? We need to demonstrate that the relations follow from the assumption of functional equivalence. If leverpressing were equivalent to searching on the patch, then rate of pressing, $B$, and rate of searching, $S$, should be related by the equation $B=c S$, the constant $c$ reflecting the difference in units between the two activities. This relation could be satisfied in two ways: (1) The proportionality $c$ could remain invariant as $B$ and $S$ changed with capture rate (rate of reinforcement), or (2) $c$ could remain constant because $B$ and $S$ remained constant across conditions, and $c$ equaled the ratio of two constants, $B / S$.

The rate of reinforcement in the chamber, $r$, is given by the equation $r=p B$, where $p$ is probability of rein- 
forcement in the random-ratio schedule. Since capture rate on the patch was approximately constant within each session (Figure 1), it is given by the equation $r=S N / A$, where $N$ is number of pellets on the patch and $A$ is the area of the patch. The time required to obtain a pellet in the chamber or on the patch would include the handling time, $H$; it would equal on the average $1 / r+H$. Since the procedure equated time per pellet in the two situations, we write:

$$
\frac{1}{p B}+H_{c}=\frac{A}{N S}+H_{p}
$$

where $H_{c}$ and $H_{p}$ are the handling times in the chamber and on the patch. $H_{c}$, the time to move to the food cup, pick up the pellet, and eat it, cannot be assumed to equal $H_{p}$, the time to poke the nose into a hole, fish out the pellet, and eat it. Substituting $c S$ for $B$ in Equation 4 and rearranging, we obtain:

$$
\frac{1}{p}=c^{\prime} \frac{1}{N}+\frac{H_{p}-H_{c}}{c S}
$$

where $c^{\prime}$ equals $A / c$. Equation 5 specifies a linear relation only if the search rate $S$ remains constant across conditions. If $H_{p}$ exceeded $H_{c}$, the $y$-intercept would be positive; if $H_{c}$ exceeded $H_{p}$, it would be negative.

Equation 5 indicates that the linear relations in Figure 2 support three conclusions. First, leverpressing was equivalent to search in that the relation $B=c S$ was satisfied. Second, the positive $y$-intercepts indicate that handling time on the patch $H_{p}$ exceeded handling time in the chamber $H_{c}$. Third, the relation $B=c S$ was satisfied because search rate $S$ and rate of pressing $B$ remained constant across conditions.

The linear cumulative records in Figure 1 indicate that the appropriate schedule to mimic work on the patch was a simple random-ratio schedule, rather than a progressive schedule. If, as these records show, the time it takes a rat to find a pellet remains about the same in a depleted patch as in a fresh patch, then no adjustment of the random-ratio schedule is needed.

The linearity of the cumulative records could arise in two possible ways: (1) the rats could have followed a systematic search pattern, which ensured that the pellets in front of them remained at the same density throughout the session (Baum, 1987), or (2) the rats' search rate within a session might have varied inversely with the density of pellets encountered (Gendron \& Staddon, 1983). Either would have tended to fix the time between pellets $-t$ in Equation 1-for a given density. If the rats searched systematically, then $D$ and $S$ in Equation 2 would have been fixed within sessions. If $S$ and $D$ varied inversely within sessions, any increase in distance between pellets would be offset by an increase in speed, with the result that the product $D S$ would tend to remain fixed. Since Equations 1,2 , and 3 assume that $t$ equals $1 / D S$, they are consistent with either possibility, because either fixing both $D$ and
$S$ or fixing their product would satisfy the requirement that $t$ be fixed for a given condition. The linear relations in Figure 2, however, favor systematic search and constancy of $S$ and $D$ within sessions because they imply that the relation $B=c S$ was satisfied by constancy of $B$ and $S$ across conditions; it seems unlikely that search rate would vary within sessions in just the right way for its average $S$ to be constant across conditions, although the possibility cannot be ruled out. Informal observation in this experiment and one by Schull et al. (1985), studying rats searching for seeds, suggested that both systematic search and compensatory changes in search rate might influence capture rate. These possibilities remain to be tested in future research.

The equivalence between leverpressing and foraging may depend on similarities between the two; both involve paws and legs to gather food. Will operant behavior that is more dissimilar to the behavior involved in foraging show such equivalence? For example, is a pigeon's keypecking equivalent to search, even though search involves legs and eyes, not a beak? Other questions remain unanswered: Under what conditions might equivalence break down? If operations can be found that result in variation in response rate and search rate, will $c$ in the equation $B=c S$ remain fixed? Further comparisons between operant behavior and foraging are needed to support the ecological validity of the operant analogue.

\section{REFERENCES}

AbarCa, N., \& Fantino, E. (1982). Choice and foraging. Journal of the Experimental Analysis of Behavior, 38, 117-123.

BAUM, W. M. (1983). Studying foraging in the psychological laboratory. In R. L. Mellgren (Ed.), Animal cognition and behavior (pp. 253283). Amsterdam: North-Holland.

BAUM, W. M. (1987). Random and systematic foraging, experimental studies of depletion, and schedules of reinforcement. In A. C. Kamil, J. R. Krebs, \& H. R. Pulliam (Eds.), Foraging behavior (pp. 587607). New York: Plenum.

BAUM, W. M. (1989). Quantitative prediction and molar description of the environment. Behavior Analyst, 12, 167-176.

Cassini, M. H., Kacelnik, A., \&egura, E. T. (1990). The tale of the screaming hairy armadillo, the guinea pig, and the marginal value theorem. Animal Behaviour, 39, 1030-1050.

Cheney, C. D., Bonem, E., Bonem, M. (1985). Changeover cost and switching between concurrent adjusting schedules. Behavioral Processes, 10, 1145-1155.

Collier, G. H., \& Rove-Collier, C. K. (1981). A comparative analysis of optimal foraging behavior: Laboratory simulations. In A. C. Kamil \& T. D. Sargent (Eds.), Foraging behavior: Ecological, ethological, and psychological approaches (pp. 39-76). New York: Garland.

FERSTER, C. B., \& SKINNER, B. F. (1957). Schedules of reinforcement. New York: Appleton-Century-Crofts.

Gendron, G. H., \& Staddon, J. E. R. (1983). Searching for cryptic prey: The effect of search rate. American Naturalist, 121, 172-186.

HANSON, J. (1987). Tests of optimal foraging using an operant analogue. In A. C. Kamil, J. R. Krebs, \& H. R. Pulliam (Eds.), Foraging behavior (pp. 335-362). New York: Plenum.

Hanson, J., \& GReen, L. (1989a). Foraging decisions: Patch choice and exploitation by pigeons. Animal Behaviour, 37, 968-986.

hanson, J., \& Green, L. (1989b). Foraging decisions: Prey choice by pigeons. Animal Behaviour, 37, 429-443. 
Hinde, R. A., \& Stevenson-Hinde, J. (Eds.) (1973). Constraints on learning. New York: Academic Press.

Holling, C. S. (1959). Some characteristics of simple types of predation and parasitism. Canadian Entomologist, 91, 385-398.

Kamil, A. C., Krebs, J. R., \& Pulliam, H. R. (Eds.) (1987). Foraging behavior. New York: Plenum.

Kamil, A. C., \& Sargent, T. D. (Eds.) (1981). Foraging behavior: Ecological, ethological, and psychological approaches. New York: Garland.

Krebs, J. R., Stephens, D. W., \& Sutherland, W. J. (1983). Perspectives in optimal foraging. In A. H. Brush \& G. A. J. Clark (Eds.), Perspectives in ornithology (pp. 165-220). Cambridge, U.K.: Cambridge University Press.

LEA, S. E. G. (1979). Foraging and reinforcement schedules in the pigeon: Optimal and nonoptimal aspects of choice. Animal Behaviour, 27, 875-886

Redhead, E., \& TYLer, P. A. (1988). An experimental analysis of op- timal foraging behaviour in patchy environments. Quarterly Joumal of Experimental Psychology, B, 40, 83-102.

SCHOENER, T. W. (1987). A brief history of optimal foraging theory. In A. C. Kamil, J. R. Krebs, \& H. R. Pulliam (Eds.), Foraging behavior (pp. 5-67). New York: Plenum.

Schull, J., Gelch, H., Vitale, J., Allan, A., James, S., \& HARRISON, M. (1985). Optimal foraging in depleting patches: Operant simulations compared with semi-natural observations. Paper presented at meetings of the Animal Behavior Society, Raleigh, NC.

SHETTLEWORTH, S. J. (1988). Foraging as operant behavior and operant behavior as foraging: What have we learned? Psychology of Learning \& Motivation, 22, 1-49.

YDENBERG, R. C. (1984). Great tits and giving up times: Decision rules for leaving patches. Behaviour, 90, 1-24.

(Manuscript received August 24, 1990; revision accepted for publication February 5, 1991.) 